\title{
Round Cell Variant of Myxoid Liposarcoma in a Japanese Macaque (Macaca fuscata)
}

\author{
H.-J Kwon, M.-S Park, D. Y. Kim, D.-Y Cho, B.-I Yoon, N.-S Shin, And D.-Y. Kim \\ Department of Veterinary Pathology, College of Veterinary Medicine, \\ Seoul National University, Seoul, Korea
}

\begin{abstract}
A 5-year-old, female, Japanese Macaque (Macaca fuscata) was diagnosed with round cell variant of myxoid liposarcoma. At necropsy, multifocal to coalescing, reddish tan to white nodules, ranging from 0.5 to $1 \mathrm{~cm}$ in diameter, were noted throughout the omentum and retroperitoneum. Similar neoplastic nodules were also present in diaphragm, abdominal wall, and on hepatic capsule. Microscopically, neoplastic masses consisted of round to polyhedral cells, which had round, often eccentric nuclei and abundant eosinophilic granular and microvacuolated cytoplasm; Oil red $\mathrm{O}$ staining demonstrated large numbers of lipid droplets in the cytoplasm. Ultrastructurally, the cytoplasm of the tumor cells was packed with occasional lipid vacuoles and numerous enlarged mitochondria. Immunohistochemistry revealed tumor cells were positive for vimentin, while negative to cytokeratin, actin, and Factor VIII-related antigen. To the authors' knowledge, this is the first report of round-cell variant of myxoid liposarcoma in nonhuman primate.
\end{abstract}

Key words: Electron microscopy; immunohistochemistry; nonhuman primate; round-cell variant of myxoid liposarcoma.

Liposarcomas are rare malignant tumors of adipose tissue. ${ }^{10,15,16}$ Cases of liposarcomas have been reported in many different animal species, with the majority reported in the dog, where the tumor is generally found in subcutis, deeper soft tissue, and thoracic and abdominal cavities. ${ }^{2,7,12}$ In humans, however, liposarcomas are most commonly found as malignant soft tissue sarcomas, accounting for approximately 16 to $18 \%$ of all soft tissue tumors 6,8 and arising commonly from extremities and retroperitoneum. ${ }^{14}$ Round-cell variant myxoid liposarcoma is one of the rarest human liposarcomas, with extremely poor prognosis. ${ }^{14}$ To our knowledge, round-cell variant myxoid liposarcoma has not been reported in a nonhuman primate. In this report, we describe the first case of round-cell variant myxoid liposarcoma arising from the abdomen of a female Japanese macaque (Macaca fuscata).

A 5-year-old female Japanese macaque was presented to the Department of Veterinary Pathology, College of Veterinary Medicine, Seoul National University, Korea for postmortem examination after a 2-week history of rapidly progressing depression, anorexia, hypothermia, and a large firm mass that could be palpable on the left retroperitoneal region. The macaque was unresponsive to fluid and symptomatic therapy and died suddenly.

At postmortem examination, multiple coalescing, tan to white, firm nodules, ranging from 0.5 to $1 \mathrm{~cm}$ in diameter could be observed throughout the omentum (Fig. 1) and the abdominal cavity contained about $50 \mathrm{ml}$ serosanguineous fluid. In the right retroperitoneum, there was a red, firm, nodular mass, $7 \mathrm{~cm}$ in diameter (Fig. 2A). On cut sections, much of the mass was composed of white to tan, multinodular masses that were firmly incorporated with the right ovary, oviduct, uterine wall, and endometrium (Fig. 2B). In addition, similar neoplastic nodules were present in the diaphragm, abdominal wall, and on the hepatic capsule.

For light microscopic examination, tissue samples of the tumor and major organs were collected, fixed in $10 \%$ neutral-buffered formalin, processed, embedded in paraffin, and stained with hematoxylin and eosin (HE). Immunohistochemistry was performed on replicate sections of the representative tumor tissues with commercially available primary antisera (DACO Corp., Carpinteria, CA) against vimentin, cytokeratin (AE1/ AE3), sarcomeric actin (AcSarco), smooth muscle actin (AcSmoothM), and Factor VIII-related antigen. The standard avidin-biotin-peroxidase technique (Vectastain kit, Vector Laboratories, Burlingame, CA) was used. For electron microscopy, the paraffin blocks were deparaffinized and rehydrated. After light microscopic examination, exact location of the tumor was diced into 1-mm cubes, postfixed in osmium tetroxide, embedded in resin (LR White Resin, London Resin Co., Ltd., Berkshire, UK), and stained with $1 \%$ phosphotungstic acid. Oil Red $\mathrm{O}$ staining was performed on frozen sections to demonstrate fat droplets in the tumor cells.

Microscopically, tumors from the omentum, ovary, uterus, liver, diaphragm, and abdominal wall had similar histologic characteristics consisting primarily of round to polygonal cells arranged in solid or loosely cellular sheets and occasionally divided by thin fibrovascular stroma (Fig. 3). The neoplastic cells had round to oval, often eccentric nuclei with 1 or 2 prominent nucleoli and abundant eosinophilic granular or micro- 

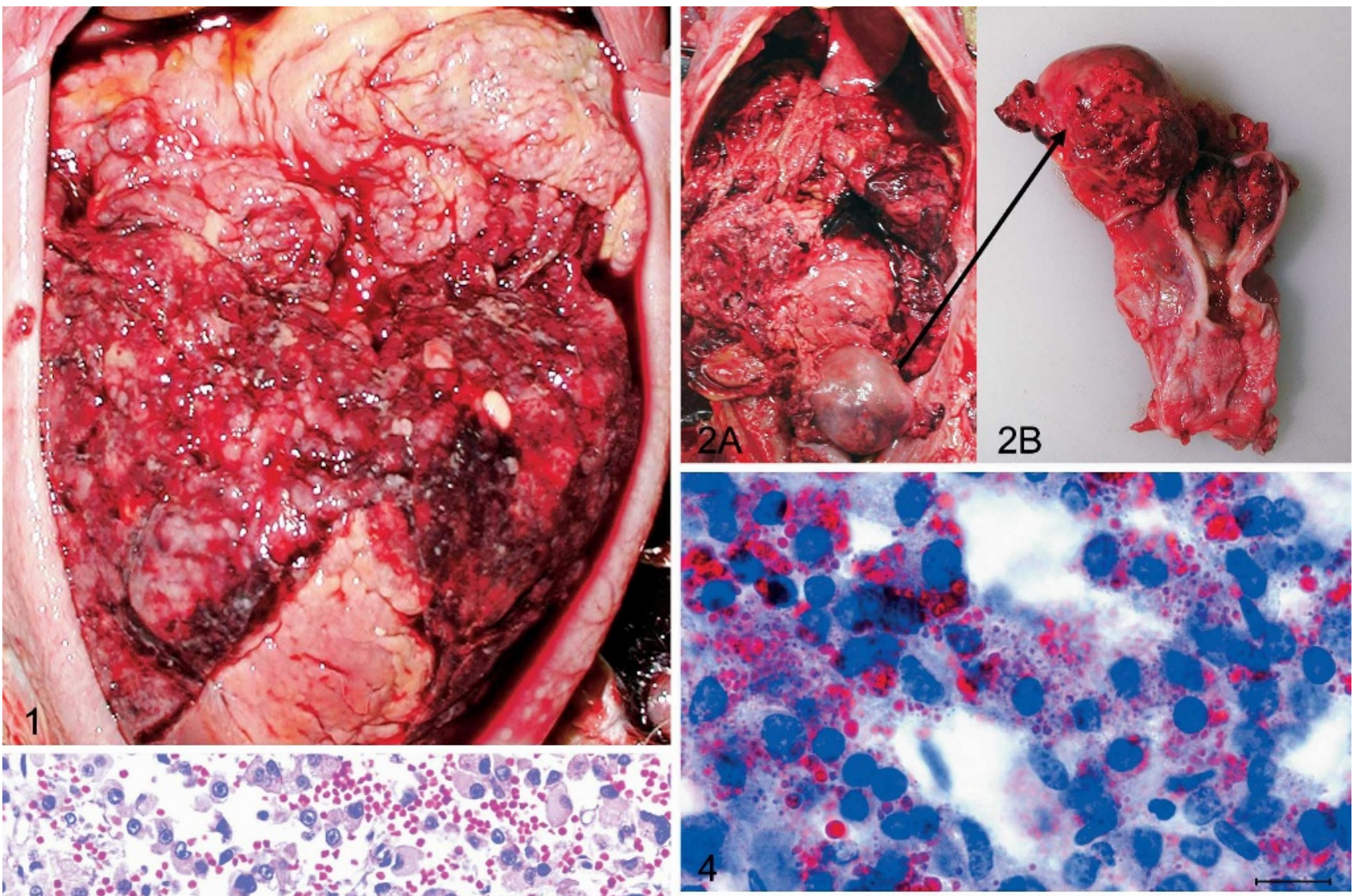

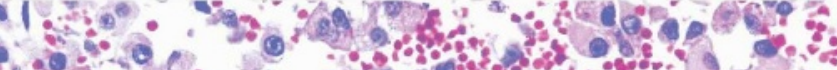

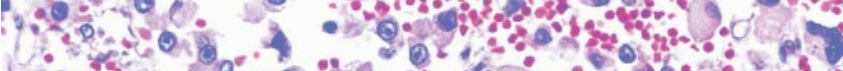

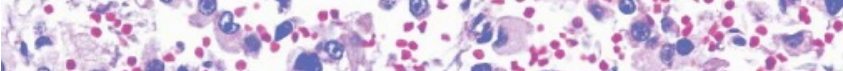

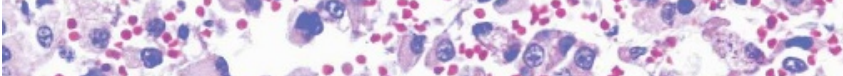

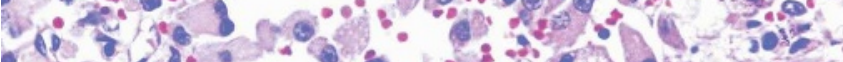

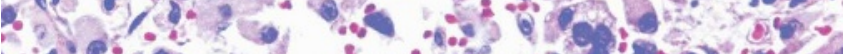

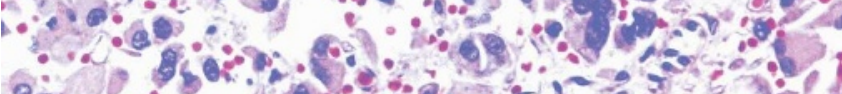

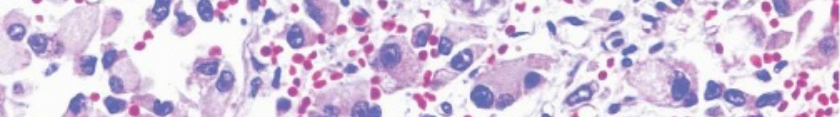

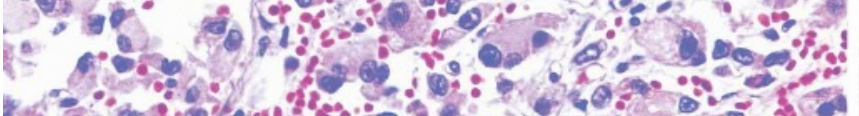

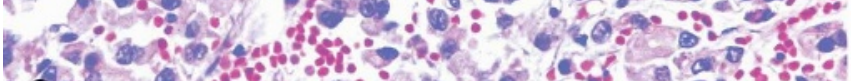
3 . ${ }^{\circ}$.

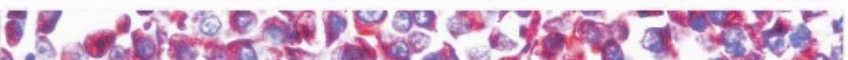

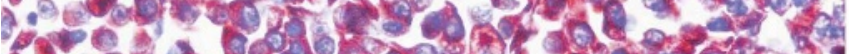

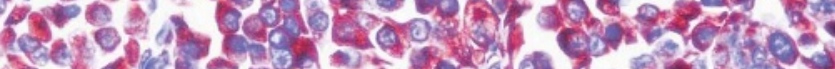

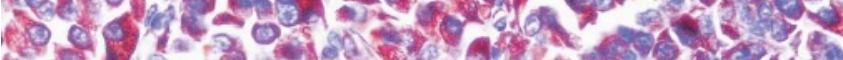

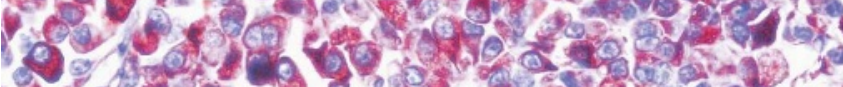

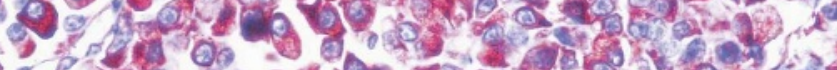

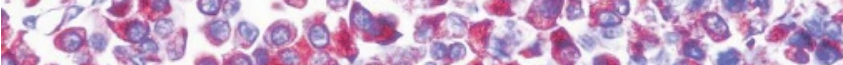

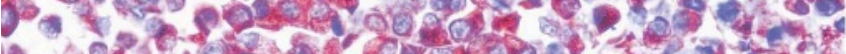
-o. 13.4 .

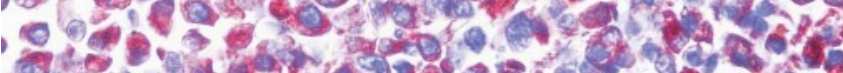

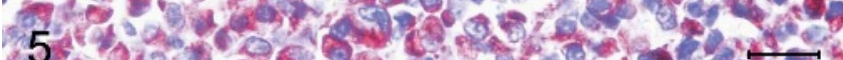

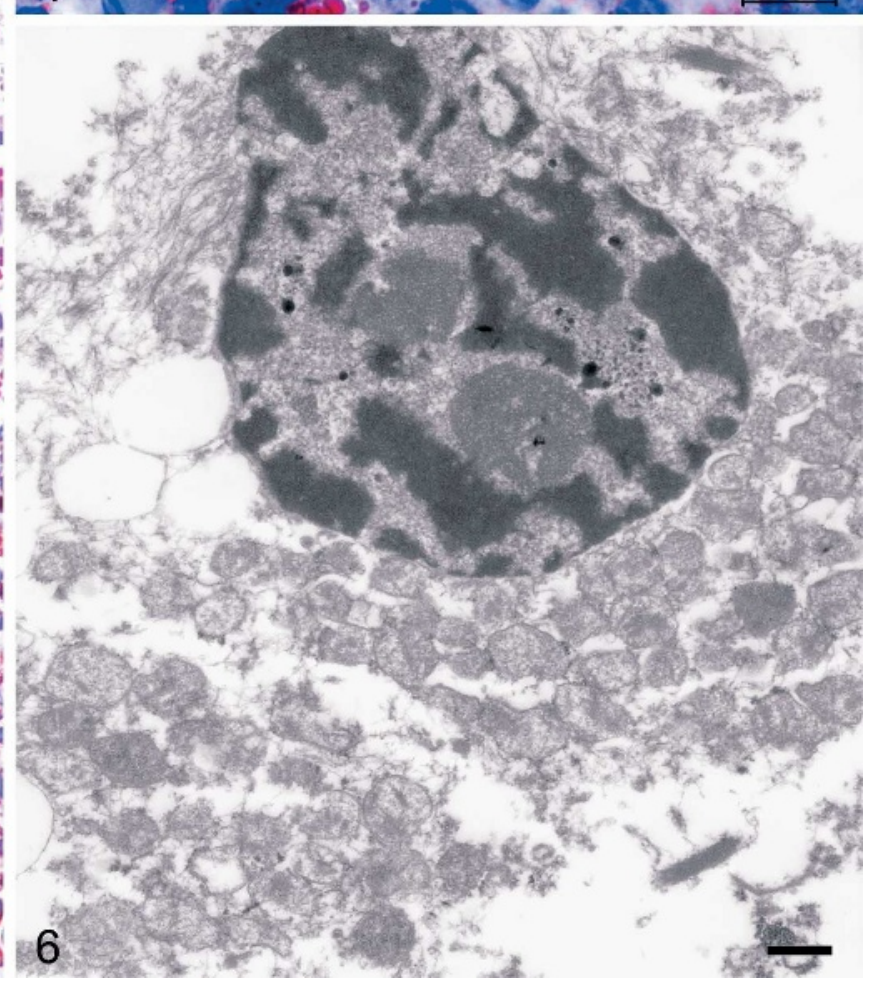

Fig. 1. Omentum; Japanese Macaque. Note multiple coalescing, tan to white, firm nodules, ranging from 0.5 to $1 \mathrm{~cm}$ in diameter observed throughout the omentum.

Fig. 2. Right retroperitoneum; Japanese Macaque. Fig. 2A. Note a red, firm, nodular mass $7 \mathrm{~cm}$ in diameter in the right retroperitoneum. Fig. 2B. On cut sections, much of the mass was composed of white to tan, multinodular masses that were firmly incorporated with the right ovary, oviduct, uterine wall, and endometrium. 
vacuolated cytoplasm. Binucleated and multinucleated tumor cells were frequently noted, whereas mitotic figures were rare. Within the tumor, there were multifocal areas of necrosis accompanied by hemorrhage and mild to moderate neutrophilic and lymphoplasmacytic inflammation. The tumor cells were implanted on the diaphragm and uterus and were infiltrated into the underlying muscle layer and myometrium, and endometrium. In the liver, the tumor cells were limited on the hepatic capsule without invasion into the parenchyma.

Oil Red O staining revealed that the cytoplasm of neoplastic cells often contained variable numbers of small lipid droplets (Fig. 4). Immunohistochemically, the neoplastic cells were intensely positive for vimentin (Fig. 5), while they were uniformly negative to the AcSarco, AcSmoothM, Factor VIII-related antigen, and AE1/AE3. Transmission electron microscopy (TEM) demonstrated the presence of numerous enlarged mitochondria and non-membrane-bound lipid vacuoles in the cytoplasm of the tumor cells (Fig. 6). In general, routine TEM reveals slightly electron-dense lipid droplets; however, in the present case, the lipid droplets were electron-lucent, because the lipid in the specimen, which was prepared from the paraffin block, was dissolved due to various solvents used during the preparation process.

Based on the gross and microscopic findings, and the results of immunohistochemistry and electron microscopy, the abdominal tumor was diagnosed as a roundcell variant myxoid liposarcoma. According to the most recent World Heath Organization (WHO) classification, human liposarcomas can be subgrouped into 4 types: well-differentiated, myxoid, pleomorphic, and dedifferentiated. ${ }^{14}$ Before the recent WHO tumor classification, round-cell liposarcoma was a distinct type of human liposarcoma, but now it belongs to the subtype of myxoid liposarcoma because myxoid and round types often form a histologic continuum. Presence of the round-cell component is associated with a more aggressive clinical course and therefore a significantly worse prognosis.

After gross examination, initial top differential diagnoses were carcinomatosis of either uterine adenocar- cinoma or ovarian carcinoma and mesothelioma because of the widespread intraperitoneal neoplastic implantation and severe involvement of the reproductive system. With microscopic examination, carcinomatosis was excluded based on the sarcoma-like morphologic characteristics and, ultimately, the negative immunoreactivity to cytokeratin and positive result to vimentin. Undifferentiated hemangiosarcoma was considered first but was soon ruled out because the tumor cells were immunohistochemically negative to the Factor VIIIrelated antigen. Cytoplasmic microvacuolization and stroma can be observed in rhabdomyosarcomas, mainly in the embryonal type. ${ }^{19}$ However, the negative results of the immunohistochemistry for AcSarco and AcSmoothM ruled out the possibility of muscle cell origin. Mesothelioma was discriminated based on the immunohistochemistry and electron microscopy results. Neoplastic cells of the malignant mesothelioma generally express both cytokeratin and vimentin. In addition, ultrastructurally, they have well-developed surface microvilli, ${ }^{17}$ which were absent in this Japanese macaque.

Ultrastructural finding of the abundant mitochondria in the tumor cells is a unique feature of this macaque. Differentials for tumors containing abundant mitochondria include oncocytomas, hibernomas, and special types of liposarcomas. Oncocytomas are well known benign tumors with atypical proliferation of mitochondria and occur in various locations including the larynx, salivary gland, lacrimal sac, and kidney. ${ }^{20}$ However, unlike the tumor cells of this particular case, the tumor cells of oncocytomas do not contain lipid droplets in the cytoplasm. Hibernomas are benign tumors arising from brown fat, ${ }^{9}$ the immature adipose tissue commonly seen in fetuses. Ultrastructural feature of hibernomas is that mitochondria are not only densely packed within the cytoplasm but also somewhat pleomorphic in contour with dense matrix as well as narrowly spaced cristae, ${ }^{9}$ which were not observed in this Japanese macaque. Moreover, hibernoma cells (multilocular adipocyte) were not seen in this case. Among liposarcomas, similar characteristic ultrastructural findings (abundant enlarged mitochondria and lipid droplets) have been described in very specific types of liposarcomas in

Fig. 3. Omentum; Japanese Macaque. Photomicrograph of a section of omental tumor nodule. Note the neoplastic cells having round to oval, often eccentric nuclei with 1 or 2 prominent nucleoli and abundant eosinophilic granular or microvacuolated cytoplasm. There was a considerable number of binucleated and multinucleated tumor cells. HE stain. Bar $=30 \mu \mathrm{m}$.

Fig. 4. Omentum; Japanese Macaque. The cytoplasm of neoplastic cells often contained variable numbers of small lipid droplets. Oil red $\mathrm{O}$ staining. Bar $=15 \mu \mathrm{m}$.

Fig. 5. Omentum; Japanese Macaque. Immunohistochemically, the neoplastic cells were intensely positive for vimentin. Avidin-biotin peroxidase complex method, hematoxylin counterstain. Bar $=30 \mu \mathrm{m}$.

Fig. 6. Omentum; Japanese Macaque. Transmission electron microscopy demonstrated the presence of numerous enlarged mitochondria and non-membrane-bound lipid vacuoles (arrow head) in the cytoplasm of the tumor cells. Bar $=0.5 \mu \mathrm{m}$. 
humans: epithelioid variant of pleomorphic liposarcoma $^{11}$ and round-cell liposarcoma originated from omentum, ${ }^{1,13}$ both of which are very rare.

Spontaneous malignant neoplasms are seldom reported in nonhuman primates; furthermore, mesenchymal-origin malignant tumors are very rare. According to a postmortem study, ${ }^{18}$ only $0.37 \%$ of the adult nonhuman primates $(4 / 1,065)$ had spontaneous malignant neoplasms (ovarian adenocarcinoma, cholangiohepatocellular carcinoma, enteric adenocarcinoma, and renal adenocarcinoma). However, 5-year culminated necropsy data of an aging colony of rhesus macaques (Macaca mulatta) showed that $30 \%$ of the deaths were related to malignant neoplasms $(26 \%$ were enteric adenocarcinomas and the remaining 4\%, various malignant tumors), ${ }^{3}$ none of which were derived from the mesenchymal tissue. Although previous studies have documented myeloliposarcomas in pottos (Perodicticus potto), ${ }^{4,5}$ to our knowledge, this is the first report of round-cell variant of myxoid liposarcoma in a nonhuman primate.

\section{Acknowledgement}

This study was supported through Brain Korea 21 Program for Veterinary Science.

\section{References}

1 Alameda F, Corominas JM, Barranco C, Munne A, Baro T, Baixeras N, Lloreta J, Serrano S: Primitive round cell liposarcoma of the omentum: diagnostic value of ultrastructural study. Ultrastruct Pathol 27:433-437, 2003

2 Baez JL, Hendrick MJ, Shofer FS, Goldkamp C, Sorenmo KU: Liposarcomas in dogs: 56 cases (1989-2000). J Am Vet Med Assoc 224:887-891, 2004

3 Beam SL: Combined-type osteosarcoma in a Rhesus macaque. Vet Pathol 42:374-377, 2005

4 Bingham G, Sembrat R, Migaki G: Myeloliposarcoma in a potto (Perodicticus potto): a case report. Lab Anim Sci 26:473-477, 1976

5 Cowgill UM: A myeloliposarcoma in a female Perodicticus potto: mineralogical and elemental chemical analysis. J Med Primatol 6:114-118, 1977

6 Daugaard S: Current soft-tissue sarcoma classifications. Eur J Cancer 40:543-548, 2004

7 Doster AR, Tomlinson MJ, Mahaffey EA, Jordan CW: Canine liposarcoma. Vet Pathol 23:84-87, 1986

8 Enzinger FM, Weiss SW: Soft tissue tumor. In: Mosby-Year Book, 3rd ed., pp. 431-466. Mosby, St. Louis, MO, 1995
9 Gaffney EF, Hargreaves HK, Semple E, Vellios F: Hibernoma: distinctive light and electron microscopic features and relationship to brown adipose tissue. Hum Pathol 14:677-687, 1983

10 Goldschmidt MH, Hendrick MJ: Tumors of the skin and soft tissues. In: Tumors in Domestic Animals, ed. Meuten DJ, 4th ed., pp. 45-117. Iowa State University Press, Ames, IA, 2002

11 Huang HY, Antonescu CR: Epithelioid variant of pleomorphic liposarcoma: a comparative immunohistochemical and ultrastructural analysis of six cases with emphasis on overlapping features with epithelial malignancies. Ultrastruct Pathol 26: 299-308, 2002

12 Messick JB, Radin MJ: Cytologic, histologic, and ultrastructural characteristics of a canine myxoid liposarcoma. Vet Pathol 26:520-522, 1989

13 Ohi M, Yutani C, Shimomukai H, Nishikawa M, Kishikawa N, Kuroda K, Nakayama M: Primary round cell liposarcoma of the omentum: a case report. Acta Cytol 36:722-726, 1992

14 Peterson JJ, Kransdorf MJ, Bancroft LW, O’Connor MI: Malignant fatty tumors: classification, clinical course, imaging appearance and treatment. Skeletal Radiol 32:493-503, 2003

15 Piercy DW, Furlong SJ, Rudram N: Multicentric liposarcoma in a cow. Vet Rec 134:310-311, 1994

16 Raubenheimer EJ, van Heerden J, Keffen RH, Lemmer LB: Liposarcoma of bone marrow origin in a kudu (Tragelaphus strepsiceros). J Wildl Dis 26:271-274, 1990

17 Sato T, Miyoshi T, Shibuya H, Fujikura J, Koie H, Miyazaki Y: Peritoneal biphasic mesothelioma in a dog. J Vet Med A Physiol Pathol Clin Med 52:22-25, 2005

18 Seibold HR, Wolf RH: Neoplasms and proliferative lesions in 1065 nonhuman primate necropsies. Lab Anim Sci 23:533-539, 1973

19 Willen H, Akerman M, Carlen B: Fine needle aspiration (FNA) in the diagnosis of soft tissue tumours: a review of 22 years experience. Cytopathology 6:236-247, 1995

20 Wilson DW, Dungworth DL: Tumors of the respiratory tract. In: Tumors in Domestic Animals, ed. Meuten DJ, 4th ed., pp. 365-399. Iowa State University Press, Ames, IA, 2002

Request reprints from D.-Y. Kim, Department of Veterinary Pathology, College of Veterinary Medicine, Seoul National University, San 56-1, Shillim-dong, Kwanak-gu, Seoul 151-742 (Korea). E-mail: daeyong@ snu.ac.kr. 